\title{
Efficacy of the Kombucha Beverage Derived from Green, Black, and Pu'er Teas on Chemical Profile and Antioxidant Activity
}

\author{
Yi Hsieh, Ming-Chung Chiu $(D$, and Jui-Yu Chou $(\mathbb{D}$ \\ Department of Biology, National Changhua University of Education, Changhua 500, Taiwan \\ Correspondence should be addressed to Jui-Yu Chou; jackyjau@cc.ncue.edu.tw
}

Received 19 July 2021; Revised 13 October 2021; Accepted 26 October 2021; Published 8 November 2021

Academic Editor: Encarna Aguayo

Copyright (c) 2021 Yi Hsieh et al. This is an open access article distributed under the Creative Commons Attribution License, which permits unrestricted use, distribution, and reproduction in any medium, provided the original work is properly cited.

\begin{abstract}
Kombucha is a symbiotic culture of bacteria and yeast which produces a high-end fruity fitness beverage by fermentation of normal tea for approximately two weeks. In addition to the advantage of being a low-sugar probiotic-rich drink, kombucha also offers the benefits of the tea used for its preparation, especially its high antioxidant content. In this research, kombucha tea was prepared by using three different tea types: green, black, and pu'er tea, and the chemical profiles and antioxidant activity were analyzed during the fermentation process (up to 20 days). The results showed that the tea type has an obvious influence on the factors associated with the antioxidant potential. The fermentation process caused a marked increase in polyphenol content and antioxidant activity initially, but this slowed progressively over time. In contrast, the fermentation of black tea contributed to the degradation of flavonoids but showed no significant effect on the other tea types. Therefore, we conclude that the tea type selected to make kombucha affects the end product as well as the fermentation time.
\end{abstract}

\section{Introduction}

Fermentation is a process that involves the breakdown of sugars by bacteria and yeast and helps to enhance food preservation. Fermenting of foods or beverages can also boost the number of beneficial bacteria or so-called probiotics. Kombucha (tea mushroom or tea fungus) is a beverage traditionally produced using a home-scale fermentation technique, made by adding a symbiotic culture of bacteria and yeast (SCOBY) to a solution of tea and sugar, a process referred to as backslopping. Kombucha has a distinctive taste which is a perfect balance between acidic, fragrant, and fruity. With the ever-increasing interest in the functional food movement and research into gut health, kombucha has gained rapid popularity around the world $[1,2]$. The beneficial effects of kombucha tea, such as its antimicrobial, antioxidant, anticarcinogenic, antidiabetic, anti-inflammatory, and cholesterol reducing properties, have been documented in some studies $[3,4]$. The oxidation of polyphenols during fermentation by the microbial communities existing in the SCOBY leads to the formation of compounds with health benefits for needy people [5].
There are key process parameters known to influence kombucha's bioactives and flavor quality, including fermentation time, the microbial composition of the SCOBY starter, sucrose concentration, and temperature [6-8].

The most characteristic microbes in kombucha fermentations are yeasts and acetic acid bacteria (AAB). Lactic acid bacteria (LAB) are usually not detected in kombucha, but addition of LAB enhances its biological functions under laboratory conditions [9]. Several symbiotic and competitive relationships exist within the kombucha microbial ecosystem. When the sucrose substrate for a kombucha fermentation is hydrolyzed into glucose and fructose by an extracellular invertase enzyme secreted by yeast cells, the concentrations of glucose and fructose increase. The acetic acid bacteria convert this glucose into gluconic acid and glucuronic acid and a low $\mathrm{pH}$ environment results. Yeasts appear to stimulate the production of glucuronic acid by $\mathrm{AAB}$. Glucose and fructose are fermented by yeasts into ethanol, carbon dioxide, and glycerol, and the ethanol produced by yeasts is used by the AAB for the production of acetic acid, which can further stimulate the production of ethanol by yeasts. The acetic acid bacteria also produce a 
cellulose pellicle which leads to biofilm formation on the surface of the fermenting liquor. Thus, because of the consumption of oxygen by the microorganisms in the zoogleal mat and the liquor, a more anaerobic environment might arise with low substrate concentrations and a high enough acidity to inhibit pathogen growth. As mentioned above, it is apparent that the fermentative process will exert a considerable influence on the final microbial community and therefore on the metabolite composition of kombucha. However, few studies have investigated the influence of tea types on the dynamics of the kombucha microbial ecosystem throughout the fermentation process as well as on the final metabolite concentrations, and further research is needed. Therefore, the purpose of this research was to understand the chemical profile and antioxidant activity of kombucha prepared from black, green, and pu'er teas at different times during the fermentation process.

\section{Materials and Methods}

2.1. Fermentation of Kombucha. Three types of leaf tea, green, black (Ten Ren Tea Co., Ltd., Taipei, Taiwan; website: https://www.tenren.com.tw), and pu'er tea from Yunnan Xiaguan Tuocha (Group) Co., Ltd., China (website: http:// www.xgtea.com) were used in this study, together with commercially available kombucha starter cultures, also from Taiwan. The starter cultures were stored in a refrigerator $\left(4^{\circ} \mathrm{C}\right.$ and $-80^{\circ} \mathrm{C}$ ) and each consisted of sour broth and a cellulosic layer (SCOBY floating on the liquid surface). The condition of fermentation was determined according to previous studies with minor modifications $[10,11]$. Sucrose $(10 \% \mathrm{w} / \mathrm{v})$ was added to deionized water just after it had boiled for $15 \mathrm{~min}$. Ten grams of tea $(10.0 \mathrm{~g} / \mathrm{L}, 1 \%)$ was added, steeped for $15 \mathrm{~min}$, and filtered using a sterile sieve [10]. The tea was then cooled to $25^{\circ} \mathrm{C}$, and $500 \mathrm{~mL}$ of tea was aliquoted into a $750 \mathrm{~mL}$ glass bottle (previously sterilized at $121^{\circ} \mathrm{C}$ for $15 \mathrm{~min}$ ). The tea broth was inoculated with $15 \mathrm{~g}$ of fresh starter culture that had been cultured in the same medium for 14 days, and the bottle was capped. Each sample was manually shaken prior to aliquoting $20 \mathrm{~mL}$ into test tubes. Three replicates were prepared for each fermentation time point and samples were completely used for measurements after each stipulated period of fermentation. Fermentation was carried out by incubating the kombucha culture at $28^{\circ} \mathrm{C}$ for 20 days and samples were analyzed every four days. Thus, a total of 18 samples for each tea type were used. The chemical profile and antioxidant activity of kombucha prepared from the different types of leaf teas were filtered and analyzed.

2.2. Total Polyphenol Content. The polyphenol content of the kombucha was measured using Folin-Ciocalteu reagent [12]. Amounts of $250 \mu \mathrm{L}$ of kombucha tea, $125 \mu \mathrm{L}$ of $50 \%$ Folin-Ciocalteu reagent (Sigma, Saint Louis, MO, USA), and $250 \mu \mathrm{L}$ of $95 \%$ ethanol were mixed, and the mixture was incubated in the dark at room temperature for $5 \mathrm{~min}$. Thereafter, $250 \mu \mathrm{L}$ of $5 \%$ sodium carbonate was added, and the mixture was incubated in the dark at room temperature for $60 \mathrm{~min}$. A blue molybdenum-tungsten complex was formed and its absorbance at $725 \mathrm{~nm}$ was measured using a microplate reader (Multiskan; ThermoFisher Scientific, Waltham, MA, USA). Total polyphenol content (TPC) was calculated by comparing the substance to standard gallic acid and was expressed as milligrams of gallic acid per milliliter of kombucha tea ( $\mathrm{mg}$ gallic acid/mL kombucha tea).

2.3. Total Flavonoid Content. Determination of total flavonoid content was conducted following Habibi et al. [13]. Appropriate dilutions of kombucha tea were mixed with sodium nitrite, then reacted with aluminum chloride. The absorbance of the solution was measured immediately at $415 \mathrm{~nm}$ using a microplate reader and the flavonoid content was measured from a calibration curve, created using quercetin solution in methanol. The total flavonoid content (TFC) was expressed as mg quercetin equivalents/mL kombucha tea.

\subsection{Antioxidant Activity Using the Ferric Ion Reducing Power} Method. A ferric ion reducing power (FRAP) assay was performed following Benzie and Strain [14], to determine the total reduction potential. An amount of $100 \mu \mathrm{L}$ of kombucha was mixed with $700 \mu \mathrm{L}$ of FRAP working solution, prepared by mixing a $10: 1: 1$ solution of $0.3 \mathrm{M}$ acetate buffer, $10 \mathrm{mM}$ TPTZ solution in $40 \mathrm{mM} \mathrm{HCl}$, and $20 \mathrm{mM}$ ferric chloride. After $60 \mathrm{~min}$ under dark conditions, the absorbance at $593 \mathrm{~nm}$ was measured using a microplate reader. The FRAP antioxidant activity was determined from a calibration curve using ferrous sulfate as a reference standard.

2.5. Antioxidant Activity Using the 2,2-Diphenyl-1-picrylhydrazyl Radical Method. A 2,2-diphenyl-1-picrylhydrazyl radical (DPPH) assay was performed according to Chu and Chen [15]. The reduction percentage of the DPPH radical was calculated to evaluate the antioxidant activity of the kombucha. The liquid layer $(250 \mu \mathrm{L})$ was mixed with $500 \mu \mathrm{L}$ of 0.2-mM DPPH solution (dissolved in methanol). The mix was vortexed and then incubated for $30 \mathrm{~min}$ at room temperature in darkness. The reaction tubes were centrifuged (13000 rpm, $1 \mathrm{~min}$ ) and $200 \mu \mathrm{L}$ samples of the supernatant were transferred into 96-well plates to measure absorbance at $517 \mathrm{~nm}$ (A517) using a microplate reader. The reduction percentage of the DPPH was calculated using the following equation:

$$
\left[\frac{1-A 517 \text { (sample) }}{A 517 \text { (blank) }}\right] \times 100 \% \text {. }
$$

2.6. Statistical Analysis. Fluctuations with incubation time of the four indicators (TPC, TFC, FRAP, and DPPH) used to quantify the chemical profile and antioxidant activity were statistically analyzed. General linear mixed models were used considering each of the indicators from the tea type to 
be the response variable, the day of incubation to be the fixed factor variable, and the experimental replication to be the random factor. The significance of the fixed factor variable was determined using a likelihood ratio test with comparison of the full model and that removed the fixed effect term. Post hoc tests were conducted using a Tukey-adjusted pairwise comparison.

To further compare the derivation efficiency of the SCOBY in different tea types with different initial chemical compounds, discrepancy of the values between the tea incubated with the SCOBY on the initial day (day 0 ) and that for 12 days of incubation were compared. Although the experiments were terminated after 20 days of incubation, the microbial community in kombucha is known to be well structured within two weeks $[10,16]$. In the present study, fluctuations in the chemical content (particularly of TFC and FRAP) also displayed an obvious turning point at 12 days of incubation (see results). Thus, we calculated the discrepancy using the following formula: $\left(V_{12}-V_{1}\right) /\left(V_{12}+V_{1}\right)$, where $V_{12}$ represents the value on day 12 and $V_{1}$ the value on day 0 . Multiple comparison of the discrepancies among the tea types was conducted using the Student's $t$-test with Bonferroni correction for $p$ values.

Correlation of fluctuations of the indicators were examined using the Spearman rank correlation. Because the biochemical fermentation processes may have slowed down by the $12^{\text {th }}$ day, correlation analysis mainly considered the values measured during the first 12 days, also showing the results for the data collected on the $16^{\text {th }}$ and $20^{\text {th }}$ day of fermentation.

The statistical analyses were conducted using the basic functions, and lme4, multcomp, and Hmisc packages in R [17].

\section{Results}

3.1. Kombucha Tea and Pellicle Appearance Varies among Teas. Kombucha tea was prepared from green, black, and pu'er teas using $1 \%(\mathrm{w} / \mathrm{v})$ tea leaves and $10 \%(\mathrm{v} / \mathrm{v})$ fermentation broth in the preparation of SCOBY as a starter culture (Figure 1(a)). After 20 days of fermentation, the surface was smooth, uneven, or with holes or bubbles. The black tea kombucha had a dark-brown color, whereas the green and pu'er tea kombuchas displayed a color somewhere between a tan brown and a creamy white. During the fermentation period of kombucha preparation over 8-12 days, a thin layer of SCOBY grew across the top of the liquid. This layer is sometimes called a pellicle and comprises a cell mass to which bacteria and yeasts are attached (Figure 1(b)). In the green tea kombucha, the structure of the pellicle was thick and flat, indicating that this treatment had the thickest surface layer. In addition, we found that this type of kombucha consistently showed an uneven SCOBY growth and formed an uneven pellicle from side-side measurements (Figures 1(c)-1(e)), with differences in wall thicknesses in different regions of up to $\sim 2-3 \mathrm{~cm}$. This uneven SCOBY growth was also observed in some of the black tea kombucha bottles.
3.2. Chemical Changes during Kombucha Tea Fermentation. During the 20 days of kombucha fermentation, the TPC in the kombucha prepared from green, black, and pu'er teas was measured every four days (Figure 2; Table 1). The initial TPC varied among the tea types and was the highest in the green tea and lowest in the pu'er tea. The three tea types showed similar fluctuations in TPC, where the amounts increased over time and stabilized after incubation for 4-8 days. However, the TPC in the green tea kombucha decreased slightly between days 12 and 16 and increased on day 20 . The effects of fermentation on TPC were greatest in the pu'er tea, followed by the black tea and the green tea (Figure 2).

In contrast to the TPC, the TFC was constant or decreased over time (Figure 3; Table 1). The initial TFC was the highest in the black tea kombucha, decreasing progressively to the lowest value on the $12^{\text {th }}$ day of fermentation, whereas it was more constant in the green and pu'er teas. The effect of fermentation on the TFC was relatively distinct in the black tea, whereas a discrepancy value close to 0 (Figure 3), indicated that fermentation scarcely affected the TFC in the green and pu'er tea kombuchas.

\subsection{Antioxidant Properties of Kombucha Teas.} Fermentation significantly improved the antioxidant activity in the kombuchas, as reflected by both indicators. The FRAP showed the highest value after fermentation for 12 days in all three tea types (Figure 4; Table 1). A sharp decline in FRAP (almost to the initial activity level) occurred between days 12 and 16 , followed by an increase on day 20 . Across the 20 -day fermentation period, the FRAP values were highest in the black tea and lowest in the green tea kombucha, whereas the SCOBY effect on FRAP was highest during fermentation of the green tea (Figure 4).

The pattern of DPPH fluctuation was similar to that of the TPC fluctuation, where the DPPH values increased over time and stabilized after fermentation for 4-8 days (Figure 5; Table 1). The DPPH value was initially the highest in the green tea kombucha, whereas the effect of fermentation on green tea DPPH was the lowest among the three tea types. The trend in DPPH values in the black and pu'er tea kombuchas was similar.

\subsection{Statistically Significant Correlation between Kombucha} Tea Parameters. Fluctuations of the values which were significantly correlated with each other are shown in Table 2 . For the values determined during the first 12 days of fermentation, fluctuations in TPC and FRAP, as well as in FRAP and DPPH, displayed positive correlative relationships in all three tea types, whereas TPC and DPPH were positively correlated for most of the tea types, with the correlation marginally significant in the pu'er tea kombucha. The TFC fluctuation was correlated with all the examined indicators only in the black tea kombucha. Most of the values measured from 16 to 20 days of fermentation were not correlated with each other. This may be caused by the different biochemical processes taking place in late fermentation, or the small number of data points, as the experiment was terminated on day 20 . 


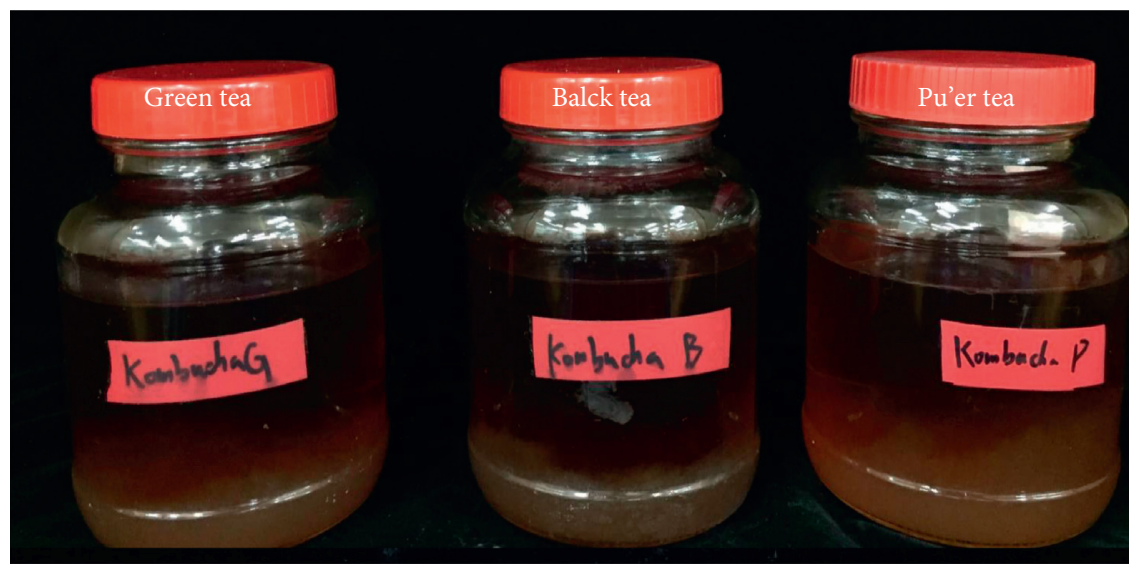

(a)

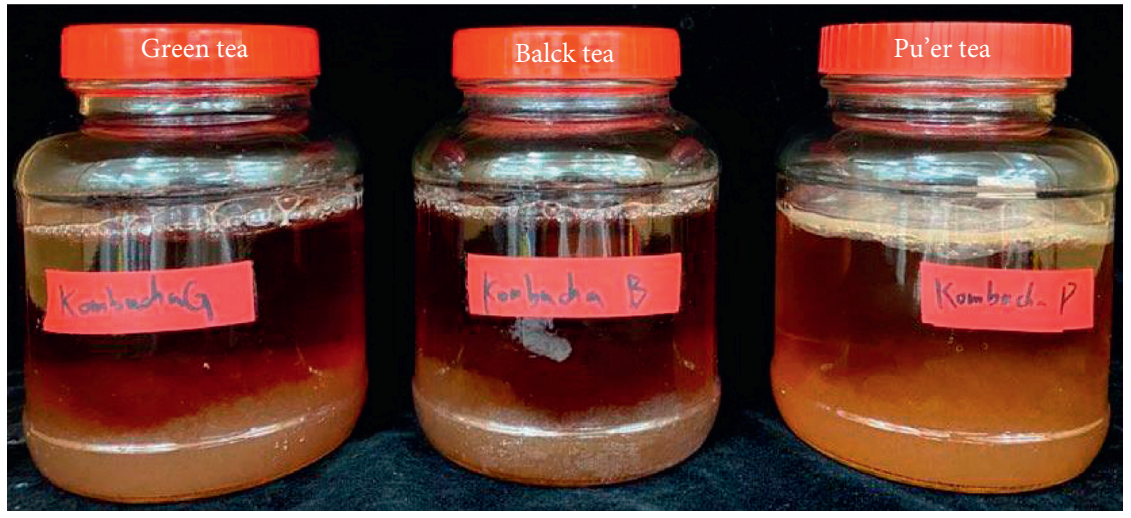

(b)

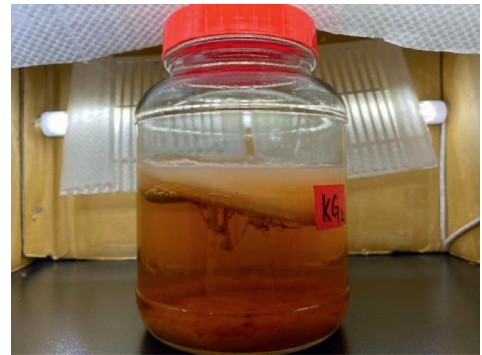

(c)

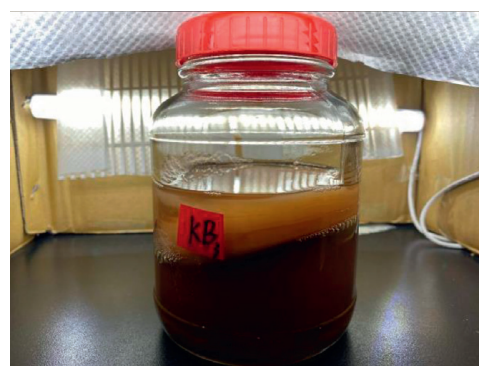

(d)

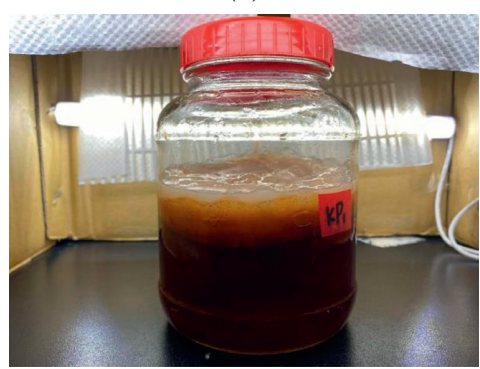

(e)

Figure 1: Appearance of kombucha prepared from green tea, black tea, and pu'er tea (from left to right) at the beginning of the fermentation process (a) and after 12 days of fermentation (b). After 20 days of fermentation, the kombucha tea prepared from black tea showed a darkbrown color, whereas the kombucha tea that was prepared from the green tea and pu'er tea displayed a color between tan brown and creamy white ((c) green tea; (d) black tea; (e) pu'er tea).
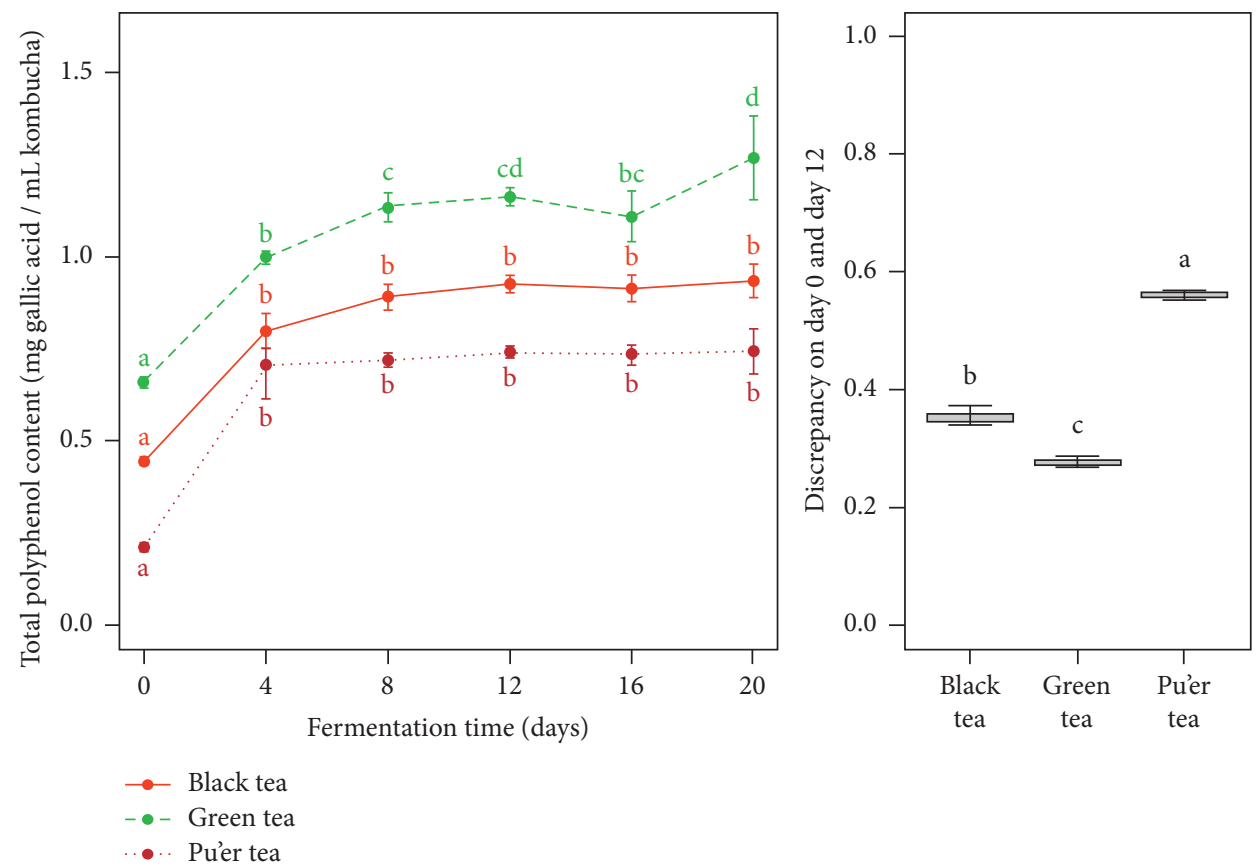

FiguRE 2: Total polyphenol content (a) and discrepancy value (b) during fermentation of kombucha prepared from green, black, and pu'er teas. Different letters indicate significant differences between the tea groups $(p<0.05)$. 
TABLE 1: The effect of fermentation time on the chemical profile and antioxidant activity of kombucha beverages produced using green, black, and pu'er teas.

\begin{tabular}{|c|c|c|c|c|}
\hline Chemical properties & Tea types & d.f. & $X^{2}$ & $p$ value \\
\hline \multicolumn{5}{|l|}{ Chemical amount } \\
\hline \multirow{3}{*}{ TPC } & Green & 5 & 52.053 & $<0.001$ \\
\hline & Black & 5 & 65.884 & $<0.001$ \\
\hline & Pu'er & 5 & 58.531 & $<0.001$ \\
\hline \multirow{3}{*}{ TFC } & Green & 5 & 19.524 & 0.002 \\
\hline & Black & 5 & 37.553 & $<0.001$ \\
\hline & Pu'er & 5 & 5.610 & 0.346 \\
\hline \multicolumn{5}{|l|}{ Antioxidant properties } \\
\hline \multirow{3}{*}{ FRAP } & Green & 5 & 36.301 & $<0.001$ \\
\hline & Black & 5 & 55.261 & $<0.001$ \\
\hline & Pu'er & 5 & 51.935 & $<0.001$ \\
\hline \multirow{3}{*}{ DPPH } & Green & 5 & 87.719 & $<0.001$ \\
\hline & Black & 5 & 73.830 & $<0.001$ \\
\hline & Pu'er & 5 & 81.981 & $<0.001$ \\
\hline
\end{tabular}

TPC: total polyphenol content; TFC: total flavonoid content; FRAP: ferric ion reducing power assay; DPPH: 2,2-diphenyl-1-picrylhydrazyl radical assay; d.f., degrees of freedom; $X^{2}$, chi square statistic for likelihood ratio test.
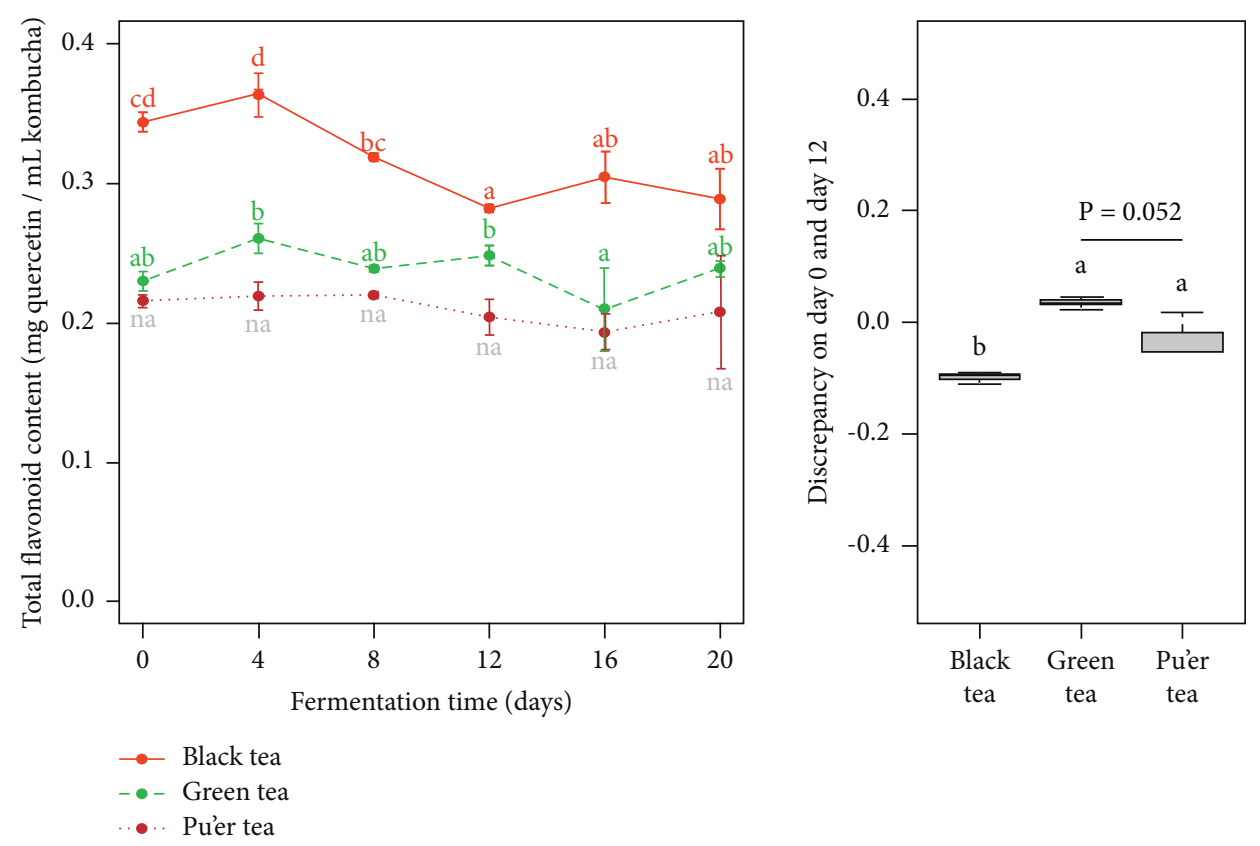

Figure 3: Total flavonoid content (a) and discrepancy value (b) during fermentation of kombucha prepared from green, black, and pu'er teas. Different letters indicate significant differences between the tea groups $(p<0.05)$.

\section{Discussion}

Fermenting beverages have traditionally been an effective way to provide flavors and vital nutrients for daily intake, and fermentation is thus rapidly rising in popularity. Kombucha stands out among such fermented beverages and its production has seen exponential growth across the globe [1]. It is usually made from black tea, but other types of kombucha prepared from different tea variants are becoming increasingly available $[10,11]$. However, many teas do not contain enough of the compounds needed to brew kombucha successfully. Previous studies have investigated the microbiological content and antibacterial properties of kombucha in detail $[3,18]$. However, there is insufficient research on the use of other tea types and their influence on kombucha health benefits and microbial dynamics. Consequently, we conducted this study which investigated kombucha production using different tea types frequently consumed in Taiwan for the preparation of kombucha and analyzed the chemical content and antioxidant potential in relation to the time of fermentation.

The predominant phytochemicals in beverage tea include catechins, theaflavins, and thearubigins, which are substances that contribute to the health benefits of tea consumption [19]. The polyphenol oxidase released during oxidation can initiate polymerization and oxidation of the catechins to theaflavins and thearubigins [20, 21]. In this study, the antioxidant activity was found to be positively 

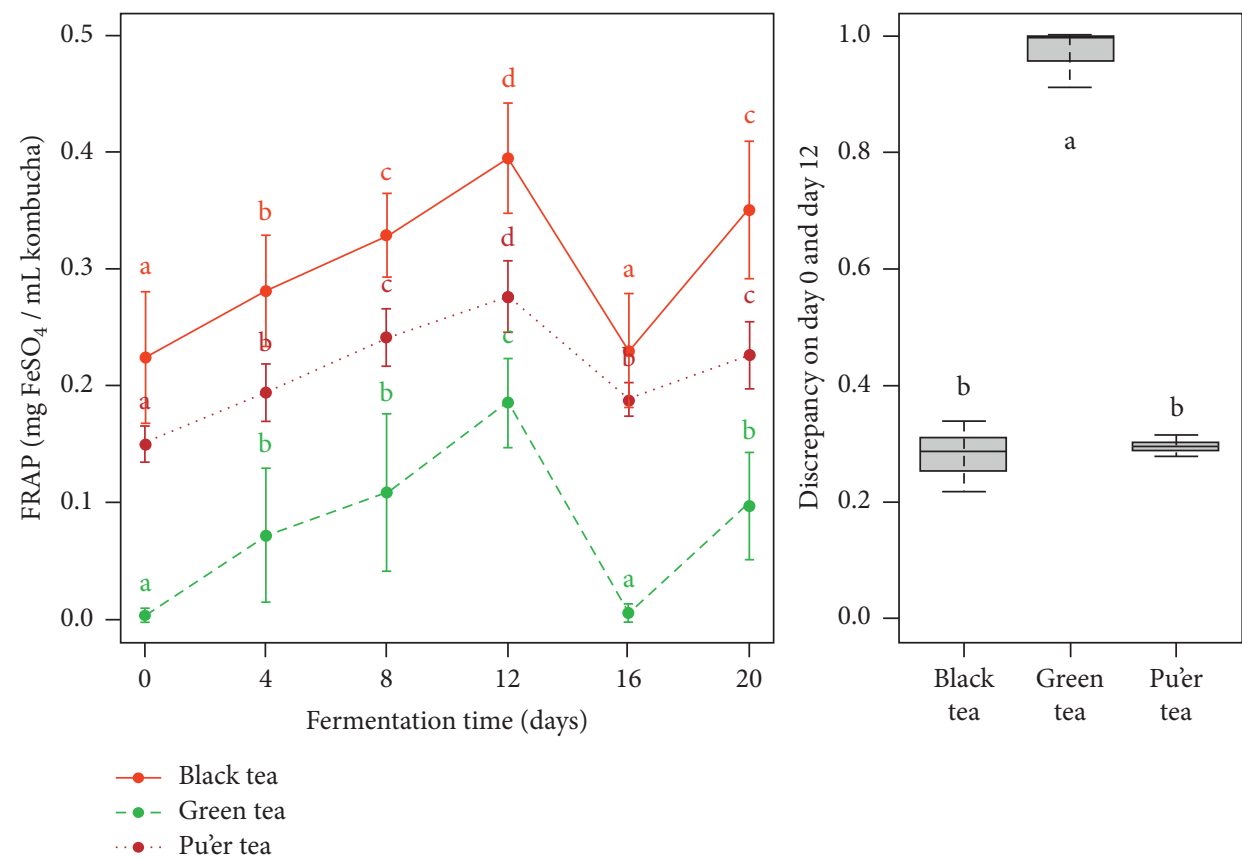

FIGURE 4: Antioxidant activity assayed using the ferric ion reducing power (FRAP) method (a) and discrepancy value (b) during fermentation of kombucha prepared from green, black, and pu'er teas. Different letters indicate significant differences between the tea groups $(p<0.05)$.
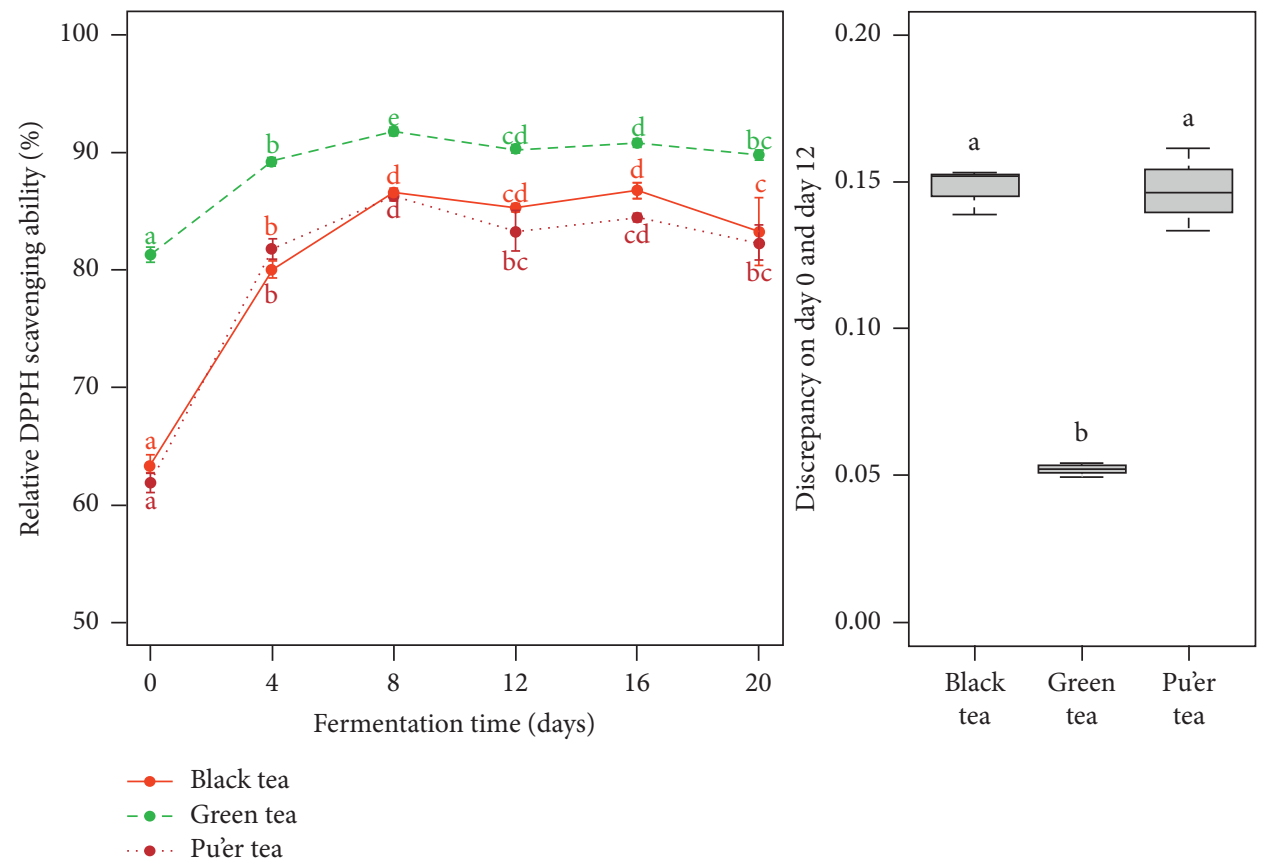

FIGURE 5: Antioxidant activity assayed using the 2,2-diphenyl-1-picrylhydrazyl radical (DPPH) method (a) and discrepancy value (b) during fermentation of kombucha prepared from green, black, and pu'er teas. Different letters indicate significant differences between the tea groups $(p<0.05)$.

related to the TPC, and this may be caused by scavenging reactive oxygen and nitrogen species and chelating redoxactive transition metal ions [22]. Despite that polyphenols could be consumed during these processes, we found that the TPC increased progressively with the duration of fermentation in all types of kombucha. The highest concentration was observed in the green tea, which also showed the highest radical scavenging activity as indicated by the DPPH values. This TPC increase could be explained by acid hydrolysis and the microbial biotransformation of condensed phenolic components [23, 24]. Prior to fermentation, the green tea was found to possess the highest 
TABle 2: Statistically significant correlations $(r)$ between parameters determined for kombucha fermented from different types of tea.

\begin{tabular}{|c|c|c|c|}
\hline Kombucha & Green tea & Black tea & Pu'er tea \\
\hline \multirow[t]{2}{*}{ TPC vs. } & $\begin{array}{c}\text { FRAP } \\
(r=0.72)^{* *} \\
\text { DPPH } \\
(r=0.79)^{* *}\end{array}$ & $\begin{array}{c}\text { Days } 0-12 \\
\text { TFC } \\
(r=-0.73)^{* *} \\
\text { FRAP } \\
(r=0.76)^{* *} \\
\text { DPPH } \\
(r=0.83)^{* *} \\
\text { Day } 12-20\end{array}$ & $\begin{array}{c}\text { FRAP } \\
(r=0.71)^{* *}\end{array}$ \\
\hline & NA & NA & NA \\
\hline \multirow[t]{2}{*}{ TFC vs. } & NA & $\begin{array}{c}\text { Days } 0-12 \\
\text { TPC } \\
(r=-0.73)^{* *} \\
\text { FRAP } \\
(r=-0.78)^{* * *} \\
\text { DPPH } \\
(r=-0.60)^{*} \\
\text { Days } 12-20\end{array}$ & NA \\
\hline & $\begin{array}{c}\text { FRAP } \\
(r=0.82)^{* * *}\end{array}$ & NA & NA \\
\hline \multirow{4}{*}{ FRAP vs. } & & Days $0-12$ & \\
\hline & $\mathrm{TPC}(r=0.72)^{* *}$ & $\mathrm{TPC}(r=0.76)^{* *}$ & $\begin{array}{c}\text { TPC } \\
(r=0.71)^{* *}\end{array}$ \\
\hline & $\begin{array}{c}\text { DPPH } \\
(r=0.69)^{*}\end{array}$ & $\begin{array}{c}\text { TFC } \\
(r=-0.78)^{* *} \\
\text { DPPH }(r=0.60)^{*} \\
\text { Days } 12-20\end{array}$ & $\begin{array}{c}\mathrm{DPPH} \\
(r=0.66)^{*}\end{array}$ \\
\hline & $\begin{array}{c}\text { TFC } \\
(r=-0.82)^{* * *}\end{array}$ & $\begin{array}{c}\mathrm{DPPH} \\
(r=-0.82)^{* *}\end{array}$ & NA \\
\hline \multirow{4}{*}{ DPPH vs. } & & Days $0-12$ & \multirow{3}{*}{$\begin{array}{c}\text { FRAP } \\
(r=0.66)^{*}\end{array}$} \\
\hline & $\mathrm{TPC}(r=0.79)^{* *}$ & $\mathrm{TPC}(r=0.83)^{* *}$ & \\
\hline & FRAP $(r=0.69)^{*}$ & $\begin{array}{c}\text { TFC }(r=-0.60)^{*} \\
\text { FRAP }(r=0.60)^{*} \\
\text { Days } 12-20\end{array}$ & \\
\hline & NA & $\begin{array}{c}\text { FRAP } \\
(r=-0.82)^{* *}\end{array}$ & NA \\
\hline
\end{tabular}

TPC: total polyphenol content; TFC: total flavonoid content; FRAP: ferric ion reducing power assay; DPPH: 2,2-diphenyl-1-picrylhydrazyl radical assay. ${ }^{*} p$ value $\leq 0.05,{ }^{* *} p$ value $\leq 0.01$, and ${ }^{* * *} p$ value $\leq 0.001$.

TPC, whereas the pu'er tea showed the lowest content of polyphenol products. Fermentation of pu'er tea also showed significantly higher efficiency in polyphenol conversion than in the other two tea groups even though it had the lowest TPC after the fermentation process. Ripened pu'er tea is processed in a special way to encourage microbial fermentation after the leaves are made by steam-compression process. Consequently, the polyphenol oxidases are inactivated by heat and the polyphenols in pu'er tea are oxidized mainly by the reaction of microorganisms [25]. It is generally believed that the older the pu'er tea, the higher its commercial value. However, it has been reported that storage time has a minor influence on phenolic compounds and antioxidant and anticancer activities of this tea [26]. Pu'er tea has attracted much attention in the field of food science due to its many bioactive constituents that have identified health benefits [27]. Thus, the development of a blend and common recipe for brewing kombucha including a combination of green and pu'er teas appears to be a worthwhile subject for future investigation.

Flavonoids are phenolic compounds and these plant secondary metabolites are widely distributed in most plant foods and tea leaves, where the levels of flavonoids vary among different types of tea. Flavonoids are considered to be strong antioxidants, and flavonoid consumption can assist humans to ward off the effects of toxins from everyday foods [28]. Studies have found that regularly drinking tea that contains these compounds is associated with reduced risk of cardiovascular disease [29]. In our study, we found flavonoids present in large quantities in the black tea but less in the green and pu'er teas. However, our findings showed that the fermentation process contributed to the degradation of this compound in the black tea, but there were no significant changes in the other two tea types. The highest concentration of flavonoids in the black tea subjected to fermentation was observed on the first day; thereafter, the flavonoid content decreased with time. This result is consistent with earlier research on kombucha tea fermentation [11, 16], and together these findings suggest that some microbial species in kombucha SCOBY are involved in the conversion of flavonoids during fermentation; however, more research is needed to determine this [30].

The different types of tea, green, black, and pu'er tea, used in this study did affect the growth of the SCOBY. During the fermentation period, the zoogleal mat formed earlier in the green tea kombucha than in that prepared from the black and pu'er teas. After 20 days of fermentation, the thickness of the floating cellulose pellicle was similar in the three types of kombucha, but that formed in the green tea kombucha consistently had an uneven thickness. Whereas the green tea showed the most significant antioxidant activity in the DPPH assay, the same tendency in DPPH activity was observed for kombucha prepared from all three tea types, indicating that the fermentation process had an impact on the increase in antioxidant properties; however, the potential decreased regardless of the tea type with duration of fermentation. Fermentation time had an effect on the decrease in reductive properties and the highest reductive potential was observed for kombucha after 8 days of fermentation. A positive correlation was also found between the reductive potential (FRAP) and polyphenol content during the first 12 days of fermentation. Thus, we also found a positive correlation between TPC and DPPH, but not in the pu'er tea kombucha. It is worth noting that, due to the differences in the antioxidant potential determined using the FRAP and DPPH methods, the green tea kombucha showed the lowest FRAP assay value, even though we found it to be the only group to show a significant effect index. The mechanisms that support antioxidant reactions with free radicals include single electron transfer (SET) and hydrogen atom abstraction (HAT). Examples of SET-based assays include DPPH and FRAP (as used in our study) in which the ionization potential of the antioxidant is the most important energetic factor in evaluating the antioxidant action [31]. The main difference between these two methods is that the former uses the free electron transfer reaction, but the latter 
utilizes metal ions for oxidation. It has been reported that high-pigmented and hydrophilic antioxidants are better reflected by ABTS, a HAT-based assay, than by the DPPH method [32]. In contrast, the FRAP method is basically a hydrophilic antioxidant assay and does not respond to lipophilic antioxidants very well [33]. Furthermore, the FRAP assay does not detect antioxidant compounds containing functional group [34]. Thus, in several studies where antioxidant activity of kombucha beverages derived from different tea types has been determined, varying relationships have been observed between the values derived using these two methods $[11,16,35]$. Therefore, further study is needed to compare the efficiency of these assays to estimate antioxidant activities and their correlations with the presence and amounts of compounds (e.g., total phenolics) involved during kombucha fermentation. Finally, it appeared to have a growth in fluctuations between the days 12 and 20 in the results of the FRAP assay. Similar findings were also revealed by other researchers [11]. As we mentioned earlier about TPC results, these fluctuations could be explained by the microbial biotransformation of related components. Further identification and research on high-throughput sequencing of microbial community during the fermentation can help to figure out the mechanisms behind these changes.

\section{Conclusions}

From the present study, we can conclude that the tea type used to make kombucha influences the end product as well as the fermentation time. The obtained findings showed differences in the chemical profile and antioxidant activity of the kombucha beverage derived from green, black, and pu'er teas. The results have shown that the fermentation process caused a marked increase in polyphenol content and antioxidant activity initially, but this slowed progressively over time. However, the fermentation of black tea contributed to the degradation of flavonoids but showed no significant effect on the other tea types. Therefore, this type of study will be useful for industry to promote probiotic beverage products.

\section{Data Availability}

The data used to support the findings of this study are available from the corresponding author upon request.

\section{Conflicts of Interest}

All authors have no conflicts of interest to report.

\section{Authors' Contributions}

Yi Hsieh and Jui-Yu Chou designed the study. Yi Hsieh and Jui-Yu Chou carried out the sample collection and experiments. Yi Hsieh, Ming-Chung Chiu, and Jui-Yu Chou conducted the data analysis. Yi Hsieh, Ming-Chung Chiu, and Jui-Yu Chou wrote the final version of the manuscript. All authors read and approved the final version of the manuscript.

\section{Acknowledgments}

The authors thank members of the Jui-Yu Chou Laboratory for comments on the manuscript and helpful discussions. This manuscript was edited by wordvice.com.tw. This work was supported by grants from the Ministry of Science and Technology, Taiwan (MOST 109-2811-B-018-500 and 1102811-B-018-001 to Ming-Chung Chiu; MOST 108-2621-B018-002-MY3 to J.-Y. Chou).

\section{References}

[1] J. M. Kapp and W. Sumner, "Kombucha: a systematic review of the empirical evidence of human health benefit," Annals of Epidemiology, vol. 30, pp. 66-70, 2019.

[2] J. Kim and K. Adhikari, "Current trends in kombucha: marketing perspectives and the need for improved sensory research," Beverages, vol. 6, no. 1, p. 15, 2020.

[3] C. Dufresne and E. Farnworth, "Tea, Kombucha, and health: a review," Food Research International, vol. 33, no. 6, pp. 409-421, 2000.

[4] C. J. Greenwalt, K. H. Steinkraus, and R. A. Ledford, "Kombucha, the fermented tea: microbiology, composition, and claimed health effects," Journal of Food Protection, vol. 63, no. 7, pp. 976-981, 2000.

[5] S. A. Villarreal-Soto, S. Beaufort, J. Bouajila, J. P. Souchard, and P. Taillandier, "Understanding kombucha tea fermentation: a review," Journal of Food Science, vol. 83, no. 3, pp. 580-588, 2018.

[6] W. Goh, A. Rosma, B. Kaur, A. Fazilah, A. Karim, and R. Bhat, "Fermentation of black tea broth (Kombucha): I. Effects of sucrose concentration and fermentation time on the yield of microbial cellulose," International Food Research Journal, vol. 19, no. 1, p. 109, 2012.

[7] R. V. Malbaša, E. S. Lončar, J. S. Vitas, and J. M. ČanadanovićBrunet, "Influence of starter cultures on the antioxidant activity of kombucha beverage," Food Chemistry, vol. 127, no. 4, pp. 1727-1731, 2011.

[8] E. Lončar, M. Djurić, R. Malbaša, L. Kolarov, and M. Klašnja, "Influence of working conditions upon kombucha conducted fermentation of black tea," Food and Bioproducts Processing, vol. 84, no. 3, pp. 186-192, 2006.

[9] N. K. Nguyen, N. T. N. Dong, H. T. Nguyen, and P. H. Le, "Lactic acid bacteria: promising supplements for enhancing the biological activities of kombucha," SpringerPlus, vol. 4, no. 1, p. 91, 2015.

[10] T. Kaewkod, S. Bovonsombut, and Y. Tragoolpua, "Efficacy of kombucha obtained from green, oolong, and black teas on inhibition of pathogenic bacteria, antioxidation, and toxicity on colorectal cancer cell line," Microorganisms, vol. 7, no. 12, p. 700, 2019.

[11] F. Gaggìa, L. Baffoni, M. Galiano et al., "Kombucha beverage from green, black and rooibos teas: a comparative study looking at microbiology, chemistry and antioxidant activity," Nutrients, vol. 11, no. 1, p. 1, 2019.

[12] S. Singhatong, D. Leelarungrayub, and C. Chaiyasut, "Antioxidant and toxicity activities of Artocarpus lakoocha Roxb. heartwood extract," Journal of Medicinal Plants Research, vol. 4, no. 10, pp. 947-953, 2010.

[13] M. Habibi, M. T. Golmakani, G. Mesbahi, M. Majzoobi, and A. Farahnaky, "Ultrasound-accelerated debittering of olive fruits," Innovative Food Science \& Emerging Technologies, vol. 31, pp. 105-115, 2015. 
[14] I. F. F. Benzie and J. J. Strain, "The ferric reducing ability of plasma (FRAP) as a measure of "antioxidant power": the FRAP assay," Analytical Biochemistry, vol. 239, no. 1, pp. 70-76, 1996.

[15] S.-C. Chu and C. Chen, "Effects of origins and fermentation time on the antioxidant activities of kombucha," Food Chemistry, vol. 98, no. 3, pp. 502-507, 2006.

[16] K. Jakubczyk, J. Kałduńska, J. Kochman, and K. Janda, "Chemical profile and antioxidant activity of the kombucha beverage derived from white, green, black and red tea," Antioxidants, vol. 9, no. 5, p. 447, 2020.

[17] R. C. Team, "R: a language and environment for statistical computing," 2013.

[18] R. Jayabalan, R. V. Malbaša, E. S. Lončar, J. S. Vitas, and M. Sathishkumar, "A review on kombucha tea-microbiology, composition, fermentation, beneficial effects, toxicity, and tea fungus," Comprehensive Reviews in Food Science and Food Safety, vol. 13, no. 4, pp. 538-550, 2014.

[19] J. Gupta, Y. H. Siddique, T. Beg, G. Ara, and M. Afzal, "A review on the beneficial effects of tea polyphenols on human health," International Journal of Pharmacology, vol. 4, no. 5, pp. 314-338, 2008.

[20] K. Sharma, S. S. Bari, and H. P. Singh, "Biotransformation of tea catechins into theaflavins with immobilized polyphenol oxidase," Journal of Molecular Catalysis B: Enzymatic, vol. 56, no. 4, pp. 253-258, 2009.

[21] S. C. Opie, M. N. Clifford, and A. Robertson, "The formation of thearubigin-like substances by in-vitro polyphenol oxidasemediated fermentation of individual flavan-3-ols," Journal of the Science of Food and Agriculture, vol. 67, no. 4, pp. 501-505, 1995.

[22] B. Frei and J. V. Higdon, "Antioxidant activity of tea polyphenols in vivo: evidence from animal studies," Journal of Nutrition, vol. 133, no. 10, pp. 3275S-3284S, 2003.

[23] R. Jayabalan, P. Subathradevi, S. Marimuthu, M. Sathishkumar, and K. Swaminathan, "Changes in freeradical scavenging ability of kombucha tea during fermentation," Food Chemistry, vol. 109, no. 1, pp. 227-234, 2008.

[24] J. V. Madeira Junior, C. B. Teixeira, and G. A. Macedo, "Biotransformation and bioconversion of phenolic compounds obtainment: an overview," Critical Reviews in Biotechnology, vol. 35, no. 1, pp. 75-81, 2015.

[25] G. Xie, M. Ye, Y. Wang et al., "Characterization of pu-erh tea using chemical and metabolic profiling approaches," Journal of Agricultural and Food Chemistry, vol. 57, no. 8, pp. 3046-3054, 2009.

[26] M. Chen, Y. Zhu, H. Zhang et al., "Phenolic compounds and the biological effects of pu-erh teas with long-term storage," International Journal of Food Properties, vol. 20, no. 8, pp. 1715-1728, 2017.

[27] H.-C. Lin, C.-T. Lee, Y.-Y. Yen et al., "Systematic review and meta-analysis of anti-hyperglycaemic effects of pu-erh tea," International Journal of Food Science and Technology, vol. 54, no. 2, pp. 516-525, 2019.

[28] J. Terao, "Dietary flavonoids as antioxidants," Forum of nutrition, vol. 61, pp. 87-94, 2009.

[29] J. M. Hodgson, "Tea flavonoids and cardiovascular disease," Asia Pacific Journal of Clinical Nutrition, vol. 17, no. Suppl 1, pp. 288-290, 2008.

[30] A. Braune and M. Blaut, "Bacterial species involved in the conversion of dietary flavonoids in the human gut," Gut Microbes, vol. 7, no. 3, pp. 216-234, 2016.

[31] N. Liang and D. Kitts, "Antioxidant property of coffee components: assessment of methods that define mechanisms of action," Molecules, vol. 19, no. 11, pp. 19180-19208, 2014.
[32] A. Floegel, D.-O. Kim, S.-J. Chung, S. I. Koo, and O. K. Chun, "Comparison of ABTS/DPPH assays to measure antioxidant capacity in popular antioxidant-rich US foods," Journal of Food Composition and Analysis, vol. 24, no. 7, pp. 1043-1048, 2011.

[33] S. E. Celik, M. Ozyürek, K. Güçlü, and R. Apak, "Solvent effects on the antioxidant capacity of lipophilic and hydrophilic antioxidants measured by CUPRAC, ABTS/persulphate and FRAP methods," Talanta, vol. 81, no. 4-5, pp. 1300-1309, 2010.

[34] X. Guan, S. Jin, S. Li, K. Huang, and J. Liu, "Process optimization, characterization and antioxidant capacity of oat (Avena Sativa L.) bran oil extracted by subcritical butane extraction," Molecules, vol. 23, no. 7, p. 1546, 2018.

[35] W. C. Tan, B. J. Muhialdin, and A. S. Meor Hussin, "Influence of storage conditions on the quality, metabolites, and biological activity of soursop (Annona muricata. L.) kombucha," Frontiers in Microbiology, vol. 11, p. 2982, 2020. 